\title{
A Hybrid Intelligent Model for Enhancing Healthcare Services on Cloud Environment
}

\author{
Ahmed Abdelaziz ${ }^{1}$ \\ Department of Information Systems \\ Higher Technological Institute, \\ Cairo, Egypt
}

\author{
Ahmed S. Salama ${ }^{2}$ \\ Department of Computer and \\ Information Systems, Sadat \\ Academy for Management Sciences, \\ Cairo, Egypt
}

\author{
A.M. $\operatorname{Riad}^{3}$ \\ Department of Information Systems, \\ Faculty of Computers and \\ Information, \\ Mansoura University, Egypt
}

\begin{abstract}
Cloud computing plays a major role in addressing the challenges of healthcare services such as diagnosis of diseases, telemedicine, maximize utilization of medical resources, etc. Early detection of chronic kidney disease on cloud environment is a big challenge that healthcare providers are facing. This paper concentrates on the using of intelligent techniques such as Decision Tree, Clustering, Linear Regression, Modular Neural Network, and Back Propagation Neural Network to address this challenge. In this paper, the researchers propose a hybrid intelligent model based on cloud computing for early revealing of chronic kidney disease. Two intelligent techniques were used: linear regression and neural network. Linear regression was used to define crucial factors that have an impact on chronic kidney disease. The proposed model for early revealing of chronic kidney disease was built using Neural Network. The accuracy of proposed model is $\mathbf{9 7 . 8 \%}$. This model outperforms on the other models existed in the previous works in terms of the accuracy and precision, recall and F1 score.
\end{abstract}

Keywords-Chronic kidney disease; linear regression; neural network; cloud computing

\section{INTRODUCTION}

Healthcare plays an important role in saving people's lives. Healthcare provides modern trends such as telemedicine, diagnosis of diseases and etc. Cloud computing plays an important role in supporting health care services. It is composed of three main services as follows:

- Medical Infrastructure.

- Medical Platform.

- Medical Software.

Cloud computing allows many patients to retrieve their medical information anywhere and anytime by using different devices such as smart phone, personal computer and etc. There are many advantages of cloud computing for healthcare services that include the following:

- Usability

- Speed

- Accessibility

- Disaster Recovery

- Cost Savings
- Reliability

- Manageability

Stakeholders (patients, doctors, nurses and etc.) can access the medical cloud to get the right medical services anytime and anywhere. There are many health care challenges that are facing cloud computing such as:

- Telemedicine Applications

- Diseases Diagnosis Applications

- Management of Electronic Health record

- Execution Time of Medical Tasks

- Maximize utilization of Medical Resources

Many diseases diagnosis applications are used on cloud computing environment to enable stakeholders for taking an appropriate medical decision. Some examples of these applications are shown below:

- Decision tree is used to reveal of cervical cancer [1].

- Support vector machine is used to reveal of hepatitis disease [2].

- Naïve Bayes is used to reveal of Heart disease [3].

- Fuzzy logic is used to reveal of breast cancer [4].

- Fuzzy logic is used to diagnosis of lung cancer [5].

- Support vector machine is used to diagnosis of diabetes disease [6].

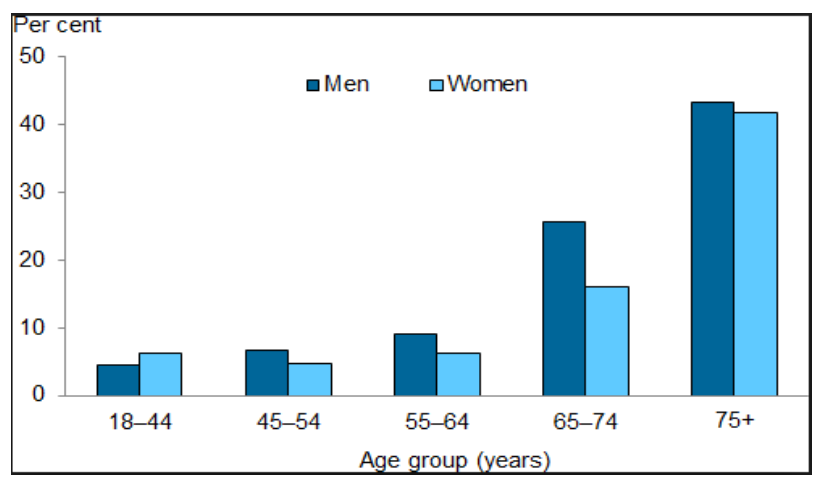

Fig. 1. Statistics of chronic kidney disease. 
Early detection of diseases is one of the most important challenges for health care services on cloud computing [7]. This paper focuses on Chronic Kidney Disease because it is one of the most serious diseases facing humanity in modern times. Many statistics indicate the growing number of Chronic Kidney patients worldwide as shown in Figure 1.

This paper introduces a hybrid intelligent model for early detection of Chronic Kidney disease on cloud computing environment by using the machine learning tool on windows azure. The rest of the paper is organized as follows: section two introduces a background overview; section three presents the related work; section four introduces the proposed intelligent model; section five introduces the experimental results; and finally, section six presents the conclusion and future work.

\section{BACKGROUND AND OVERVIEW}

This section presents a review of Chronic Kidney disease, neural network and regression analysis as follows:

\section{A. Overview of Chronic Kidney Disease}

Chronic kidney disease is considered one of the most dangerous diseases that are facing the worldwide.

TABLE. I. INITIAL FACTORS THAT INFLUENCE ON CHRONIC KIDNEY DISEASE

\begin{tabular}{|c|c|c|}
\hline No & Factor & Description \\
\hline 1 & Anemia & Nominal \\
\hline 2 & Age & Numerical \\
\hline 3 & Pedal edema & Nominal \\
\hline 4 & Blood pressure & Numerical \\
\hline 5 & Appetite & Nominal \\
\hline 6 & Specific gravity & Nominal \\
\hline 7 & Coronary artery disease & Nominal \\
\hline 8 & Albumin & Nominal \\
\hline 9 & Diabetes mellitus & Nominal \\
\hline 10 & Sugar & Nominal \\
\hline 11 & Hypertension & Nominal \\
\hline 12 & Red blood cells & Nominal \\
\hline 13 & Red blood cell count & Numerical \\
\hline 14 & Pus cell & Nominal \\
\hline 15 & White blood cell count & Numerical \\
\hline 16 & Pus cell clumps & Nominal \\
\hline 17 & Packed cell volume & Numerical \\
\hline 18 & Bacteria & Nominal \\
\hline 19 & Hemoglobin & Numerical \\
\hline 20 & Blood glucose random & Numerical \\
\hline 21 & Potassium & Numerical \\
\hline 22 & Blood urea & Numerical \\
\hline 23 & Sodium & Numerical \\
\hline 24 & Serum Creatinine & Numerical \\
\hline 25 & Class & Nominal \\
\hline
\end{tabular}

There are many factors that are influencing chronic kidney disease as shown in Table 1. A data set of chronic kidney disease from machine learning repository was used. The data set contains 800 instances.

\section{B. Overview of Linear Regression}

Linear regression has two types of regressions which are simple linear regression and multiple linear regressions. General equation of linear regression is formulated as follows:

$\mathrm{M}=\beta_{0}+\beta_{1} \mathrm{y}_{1}+\beta_{2} \mathrm{y}_{2}+\ldots \ldots . \mathrm{B}_{\mathrm{N}} \mathrm{y}_{\mathrm{N}}+\epsilon$

Where:

- $\mathbf{M}$ is the dependent variable

- $\mathrm{y}_{1}, \mathrm{y}_{2}, \mathrm{y}_{\mathrm{N}}$ are the independent variables

- $\beta_{\mathrm{i}}$ represents the regression coefficient

- $\varepsilon$ represents the random error component.

- $\beta_{0}$ represents the y intercept

In linear regression, there are two main criteria's to measure the performance of the proposed model which are coefficient of determination and feature weight of independent variables as shown in Figure 2.

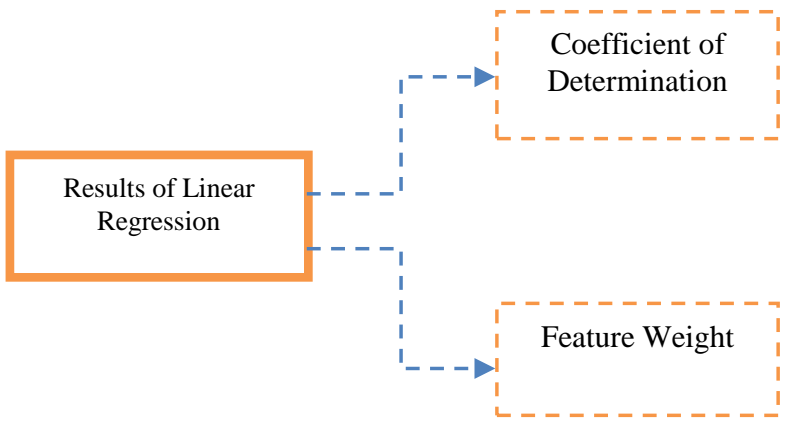

Fig. 2. Two main outputs in linear regression.

- Coefficient of determination indicates the level of performance of the linear regression model.

- Feature weight shows which variables are important. Whenever, feature weight is less than 0.05, and then the independent variable is considered significant variable.

\section{Overview of Neural Network}

Neural network is used to implement many intelligent applications such as diseases diagnosis and data classification and etc. There are some types of neural networks such as:

- Feed-forward network

- Back-propagation network

- Modular neural network

- Recurrent neural network

The neural network consists of input layers, hidden layers and output layers as shown in Figure 3. 


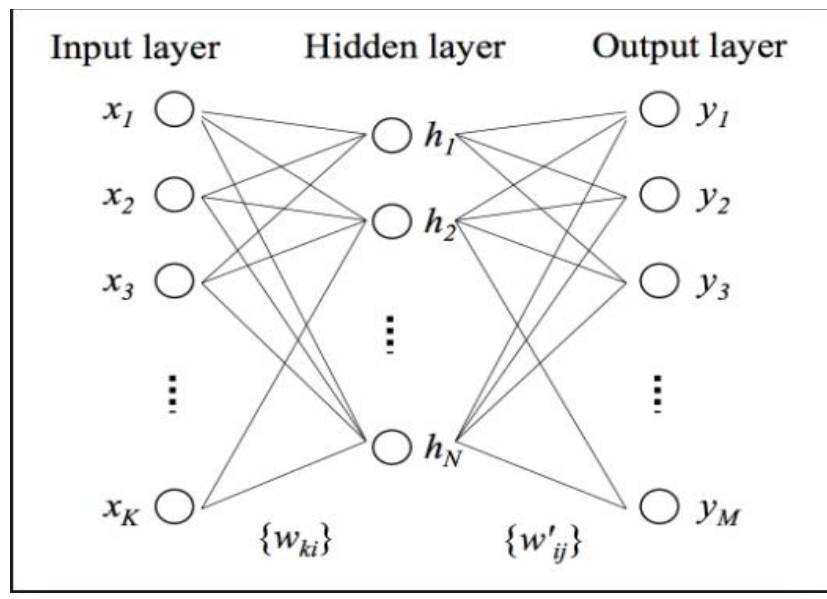

Fig. 3. General structure of neural network.

\section{RELATED WORK}

This section introduces many researches that seek to detect chronic kidney disease as follows:

Lambodar Jena and et al, presented a novel model to detect chronic kidney disease by using data mining classification. This research is used WEKA tool. The accuracy of the proposed model is $95 \%$. Naive Bayes outperforms on decision tree. The importance of the research is to predict chronic kidney disease by naive Bayes technique [8].

K. R. Anantha and et al, introduced a model which is decision tree technique for predicting chronic kidney disease on WEKA tool. This research shows that decision tree outperforms on clustering technique. The accuracy of the proposed model is $91 \%$. The importance of the research is to predict chronic kidney disease by decision tree [9].

Basma B. and et al, presented a model for detecting chronic kidney disease by using support vector machine. The results show that support vector machine better than decision tree in terms of execution time and accuracy. The accuracy of the proposed model is $95 \%$. The importance of the research is to detect chronic kidney disease by support vector machine [10].

Asif S. and et al, introduced intelligent model for revealing chronic kidney disease by using clustering technique on WEKA tool. This research shows clustering technique better than decision tree in order to accuracy. The accuracy of the clustering technique is $96 \%$. The importance of the research is to reveal chronic kidney disease by clustering technique [11].

RUEY K. C. and et al, presented an approach for detecting of chronic kidney disease on cloud computing environment. The results show that back propagation neural network outperforms on modular neural network and feed forward neural network in order to accuracy and precision. The accuracy of the proposed system is $94.7 \%$. The importance of the study is to detect chronic kidney disease on cloud environment [12].

Chien. z. W. and et al, introduced fuzzy expert system for revealing of chronic kidney disease on cloud computing environment. This research shows that fuzzy expert system better than neural network in order to accuracy and mean square error. The accuracy of the proposed model is $88.4 \%$. The importance of the study is to reveal chronic kidney disease by fuzzy expert system on cloud environment [13].

Stuti N. and et al, introduced a new model based on Naive Bayes to predict chronic kidney disease on cloud environment (Google Application Engine). The results show that Naive Bayes outperforms on neural network in order to accuracy and precision. The accuracy of the proposed model is $97.1 \%$. The importance of the research is to predict chronic kidney disease on Google Application Engine [14].

Anu B. and et al, introduced survey of many researches that seek to predict chronic kidney disease by using intelligent techniques. The importance of the research is to review many researches of chronic kidney disease [15].

Table 2 introduces summary of intelligent techniques of chronic kidney disease researches.

TABLE. II. SUMMARY OF RELATED WORK

\begin{tabular}{|c|c|c|}
\hline No & Category & Some Approach \\
\hline 1 & $\begin{array}{l}\text { intelligent techniques } \\
\text { applied of chronic kidney } \\
\text { disease }\end{array}$ & $\begin{array}{ll}> & \text { quantitative approach } \\
> & \text { Naive Bayes } \\
> & \text { mean analysis } \\
> & \text { mean time and modeling } \\
& \text { regression } \\
> & \text { time series } \\
> & \text { Clustering } \\
> & \text { Feed Forward neural } \\
& \text { network } \\
> & \text { Back Propagation neural } \\
& \text { networkl } \\
> & \text { Modular neural network } \\
> & \text { Decision tree } \\
> & \text { Support vector machine } \\
> & \text { Fuzzy expert system }\end{array}$ \\
\hline
\end{tabular}

Through related work, intelligent applications are not accurate on cloud computing environment for revealing of chronic kidney disease. So, this paper introduces a new intelligent model for revealing chronic kidney disease on cloud environment.

\section{THE PROPOSED APPROACH}

This section introduces an intelligent model to reveal Chronic Kidney disease on cloud environment. As shown in Figure 4, this intelligent model has three steps:

1) Survey recent studies related to Chronic Kidney disease to develop a candidate list of factors that influence this disease.

2) Identify the critical factors form this candidate list using linear regression.

3) Develop an intelligent model for revealing chronic kidney disease based neural network. 


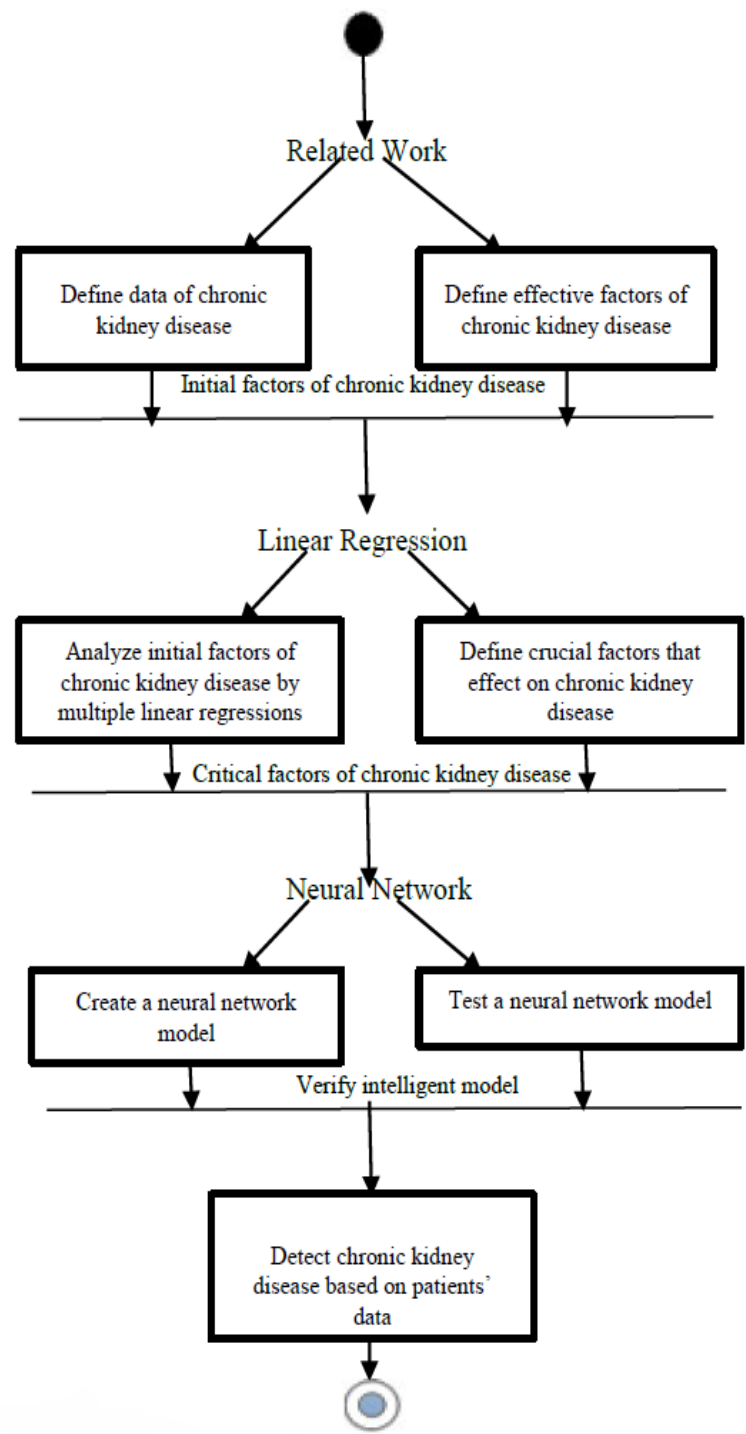

Fig. 4. The proposed model for revealing of chronic kidney disease.

\section{THE Hybrid INTELLIGENT MODEL FOR HEALTHCARE SERVICES}

This section presents the proposed hybrid model for healthcare services on cloud computing. It includes two intelligent techniques which are explained below.

\section{A. Linear Regression}

Linear regression is used to identify the critical factors of Chronic Kidney disease (CFCKD). It is composed of one dependent variable and 13 independent variables. It is formulated as follows:

$$
\mathrm{M}=\beta_{0}+\beta_{1} \mathrm{CFCKD}_{1}+\beta_{2} \mathrm{CFCKD}_{2}+\ldots \ldots . \mathrm{B}_{\mathrm{N}} \mathrm{CFCKD}_{13}+\epsilon
$$

Where:
$M$ is the dependent variable (degree of influence of candidate factors on Chronic Kidney disease) and $C F C K D_{l}$, $C_{C F C K D_{2} \ldots} C_{C F C K D_{13}}$ are the independent variables (candidate factors).

This section also introduces linear regression algorithm to identify critical factors of Chronic Kidney disease.

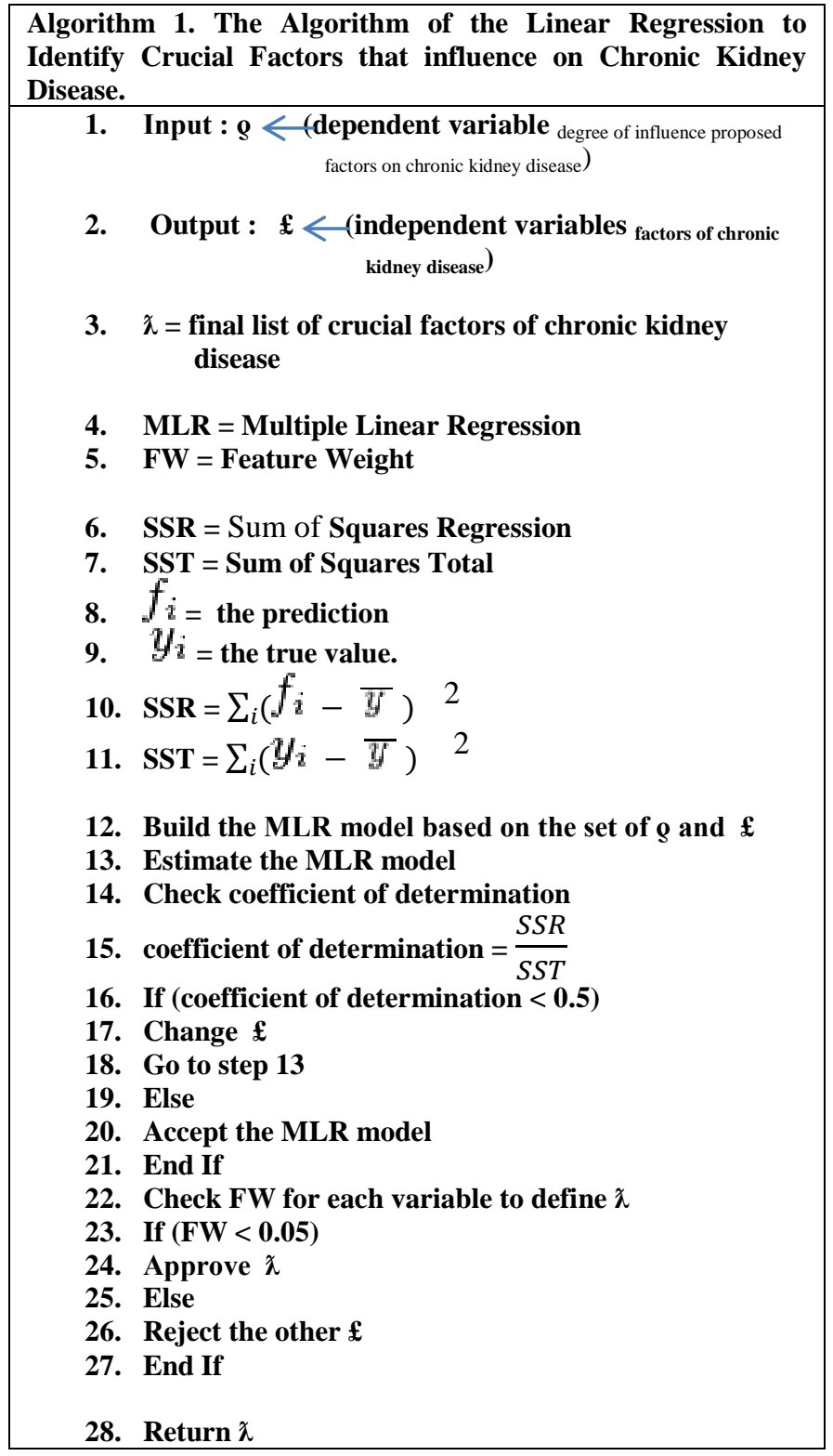

\section{B. Neural Network}

Neural network has 13 critical factors as input layer, one hidden layer and two outputs (Chronic Kidney or No Chronic Kidney).

This section introduces neural network algorithm to reveal of Chronic Kidney disease. 
TABLE. III. SUMMARY OF CRITICAL FACTORS OF CHRONIC KIDNEY

\begin{tabular}{|l|c|}
\hline Algorithm 2. The Proposed Algorithm of the Neural Network to \\
Reveal of Chronic Kidney Disease.
\end{tabular}

\section{EXPERIMENTAL RESULTS}

This section presents the execution of the proposed hybrid model on windows azure as shown in Figure 5.
DISEASE

TABLE. III. SUMMARY OF CRITICAL FACTORS OF CHRONIC KIDNEY
\begin{tabular}{|l|l|l|}
\hline No & Factor Name & FW \\
\hline 1 & Anemia & -0.002 \\
\hline 2 & White Blood Cell Count & 0.0007 \\
\hline 3 & Packed Cell Volume & -0.002 \\
\hline 4 & Hemoglobin & 0.03 \\
\hline 5 & Potassium & -0.0006 \\
\hline 6 & Sodium & 0.002 \\
\hline 7 & Serum Creatinine & -0.05 \\
\hline 8 & Blood Urea & 0.003 \\
\hline 9 & Blood Glucose Random & 0.005 \\
\hline 10 & Sugar & 0.04 \\
\hline 11 & Specific Gravity & -0.002 \\
\hline 12 & Blood Pressure & -0.00005 \\
\hline 13 & Age & 0.02 \\
\hline
\end{tabular}

Finished running

\section{Chronic_Kidney_Disease}

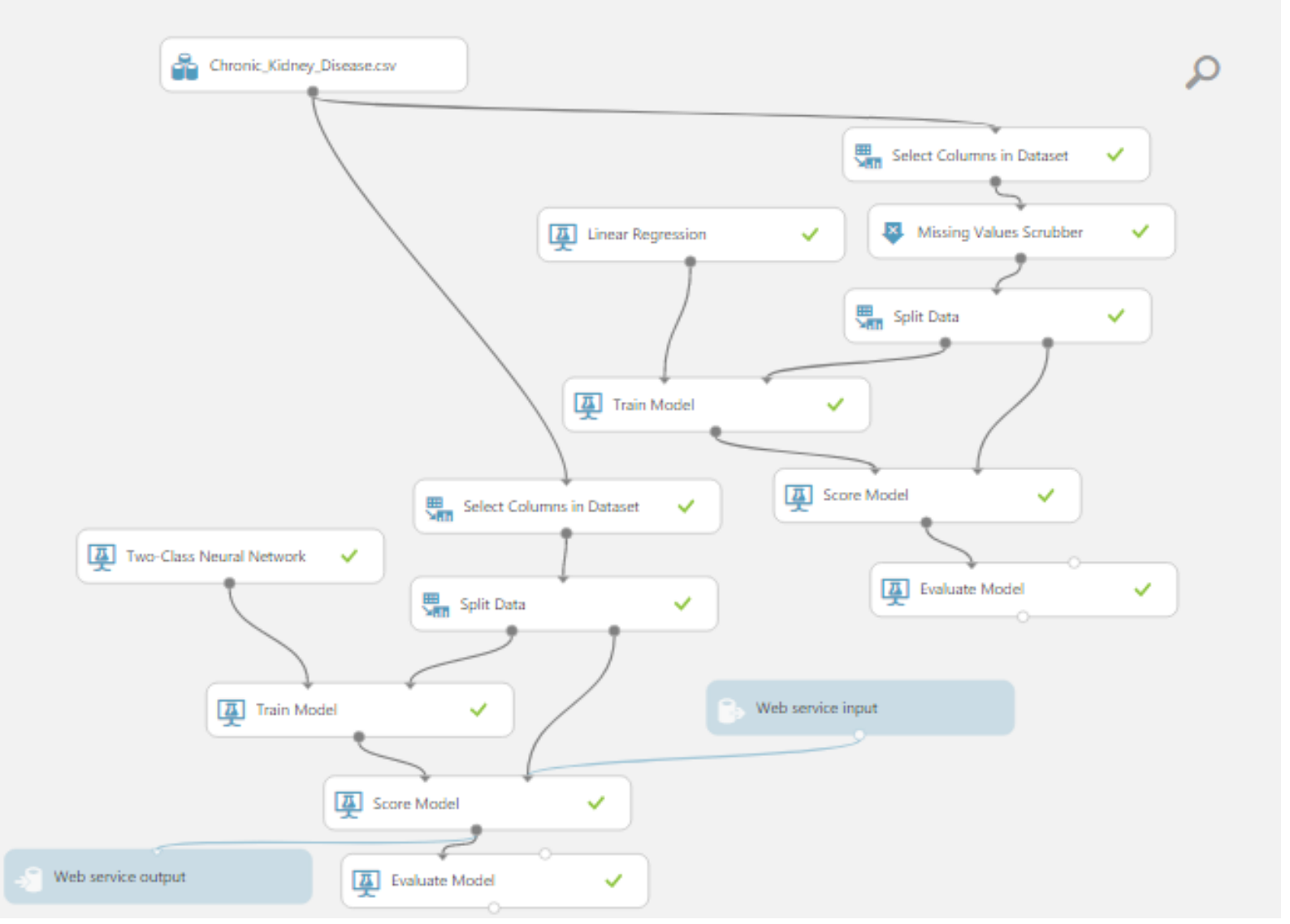

Fig. 5. Execution of hybrid model on windows azure. 


\section{A. Execution of Linear Regression on Windows Azure}

This section introduces the execution of linear regression on windows azure. Linear regression was used to define critical factors of Chronic Kidney disease. Table 3 shows the critical factors of Chronic Kidney disease via the feature weights. Whenever, feature weight is less than 0.05, the factor is medically important. For example, the feature weight for Serum Creatinine (feature weight $=-0.04$ ) is less than 0.05, then this factor is medically important as shown Chronic Kidney in figure 6.

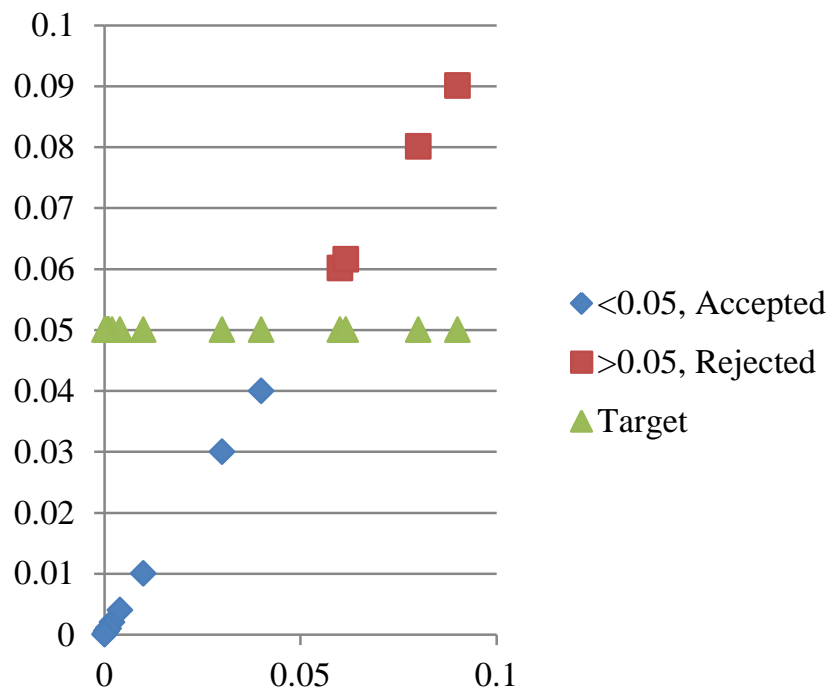

Fig. 6. Condition statement of factors of chronic kidney disease.

\section{B. Execution of Neural Network on Windows Azure}

This section introduces the execution of neural network on windows azure. NNM is composed of 13 neurons in input layer, one hidden layer and two outputs. The neural network type used in this paper is the two class neural network. Table 4 shows the performance of the neural network. It defines accuracy, precision, recall and $\mathrm{f} 1$ score. The accuracy of the proposed model is $97.8 \%$ as shown in Figure 7.
TABLE. IV. PERFORMANCE ATTRIBUTES OF NNM

\begin{tabular}{|l|l|l|l|}
\hline True Positive & False Negative & Accuracy & Precision \\
\hline 51 & 0 & 0.978 & 0.962 \\
\hline False Positive & True Negative & Recall & F1 Score \\
\hline 2 & 39 & 1.000 & 0.981 \\
\hline
\end{tabular}

\section{Case Study}

Three real cases of patients were conducted on windows azure to reveal of Chronic Kidney disease (CKD) as shown in Table 5 and Figure 8.

TABLE. V. DATA OF CKD PATIENTS

\begin{tabular}{|l|l|l|l|l|}
\hline No & Factor Name & Patient 1 & Patient 2 & Patient 3 \\
\hline 1 & Age & 49 & 59 & 29 \\
\hline 2 & Blood Pressure & 71 & 81 & 101 \\
\hline 3 & Specific Gravity & 2.007 & 1.026 & 1.2 \\
\hline 4 & Sugar & 0 & 0 & 1 \\
\hline 5 & $\begin{array}{l}\text { Blood Glucose } \\
\text { Random }\end{array}$ & 127 & 132 & 121 \\
\hline 6 & Blood Urea & 57 & 19 & 21 \\
\hline 7 & Serum Creatinine & 3.9 & 1.2 & 1.3 \\
\hline 8 & Sodium & 113 & 142 & 131 \\
\hline 9 & Potassium & 2.6 & 3.6 & 2.2 \\
\hline 10 & Hemoglobin & 12.2 & 15.9 & 11 \\
\hline 11 & $\begin{array}{l}\text { Packed Cell } \\
\text { Volume }\end{array}$ & 33 & 54 & 51 \\
\hline 12 & $\begin{array}{l}\text { White Blood Cell } \\
\text { Count }\end{array}$ & 5700 & 6900 & 5100 \\
\hline 13 & Anemia & Yes & No & Yes \\
\hline 14 & Class & CKD & NOCKD & NOCKD \\
\hline 15 & Probability & 0.00005 & 0.99993 & 0.99997 \\
\hline
\end{tabular}

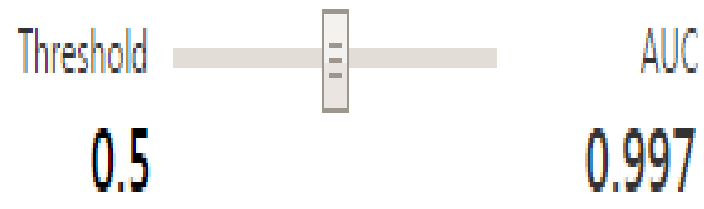

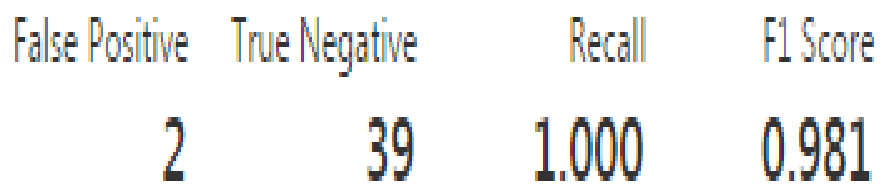

Fig. 7. Performance attributes of hybrid model on windows azure. 


\section{Probability}

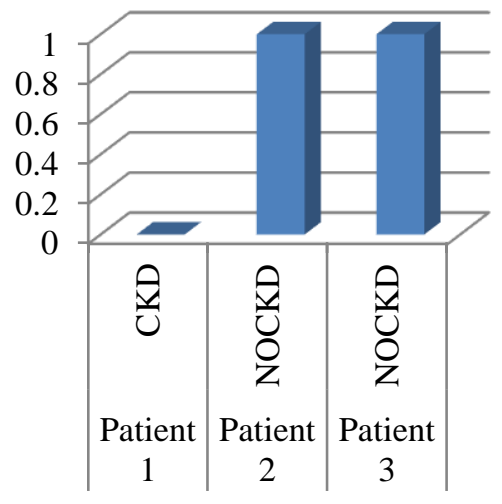

Probability

Fig. 8. Probabilities of CKD patients.

Figure 9 shows the accuracy of the neural network model on windows azure. This model outperforms on the other models existed in the previous works in terms of the accuracy and precision, recall and F1 score.

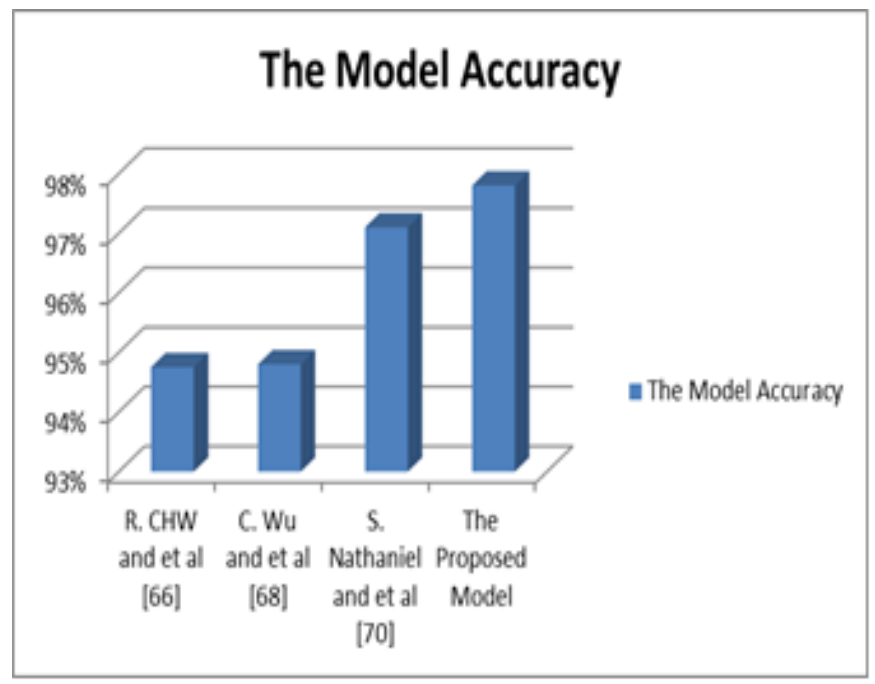

Fig. 9. Accuracy of the NNM compared to the rest of the models.

\section{CONCLUSION AND FUTURE WORK}

Cloud computing raises healthcare performance. Early detection of diseases is one of the most challenges that are facing healthcare providers. Chronic kidney disease is considered one of the dangerous diseases that are facing humanity worldwide. The accuracy of many traditional applications are not good enough for revealing chronic kidney disease. This paper tries to introduce intelligent model based on cloud computing for address this challenge. The proposed hyprid model includes two techniques: linear regression and neural network. The proposed model was implemented on windows azure. The accuracy of the proposed model is $97.8 \%$. Three real cases of patients were conducted on windows azure to emperically validate the proposed model.

It is recommeded as future work:

- To use logistic regression for determining critical factors that affect Chronic Kidney disease.

- To uses deep learning for revealing Chronic Kidney disease on cloud computing environment.

REFERENCES

[1] S. Sha "Cervical Cancer stage prediction using Decision Tree approach of Machine Learning", IJARCCE, Vol.5, No.4, 2016, pp. 345-348.

[2] C. Kum, M. V. Kumar, T. Gayathri and S. R. Kumar "Data Analysis and Prediction of Hepatitis Using Support Vector Machine (SVM)", IJCSIT, Vol.5, No.2, 2014, pp. 2235-2237.

[3] Prera. T, Shivaprakash .N and Swetha .N "Prediction of Heart Disease Using Machine Learning Algorithms- Naïve Bayes,Introduction to PAC Algorithm, Comparison of Algorithms and HDPS", IJSE, Vol.3, No.2, 2015, pp. 90-99.

[4] Tin. P and Paulin. R "Detect Breast Cancer using Fuzzy C means Techniques in Wisconsin Prognostic Breast Cancer (WPBC) Data Sets", IJCAT, Vol.2, No.5, 2013, pp. 614-617.

[5] M. HA "Lung Cancer Diagnosis by Using Fuzzy Logic", ijcsmc, Vol.5, No.3, 2016, pp. 32-41.

[6] Arjn and An. S "Diagnosis of Diabetes Using Support Vector Machine and Ensemble Learning Approach", IJEAS, Vol.2, No.11, 2015, pp. 6872 .

[7] M. Elhosen, A. Salama, Ahmed. A, A. M. Riad, "Intelligent Systems Based on Cloud Computing for Healthcare Services: A Survey", International Journal of Computational Intelligence Studies, Vol. 6, Nos. 2/3, 2017, pp. 157-188.

[8] L. Jen and N. Kam "Distributed Data Mining Classification Algorithms for Prediction of Chronic- Kidney-Disease", IJERMT, Vol.4, No.11, 2015, pp. 110-118

[9] K. Padmanab and G. Parthib "Applying Machine Learning Techniques for Predicting the Risk of Chronic Kidney Disease", ijst, Vol.9, No.29, 2016, pp. 1-5.

[10] B. Bouken, H. Mousan and A. Ha "Performance of Data Mining Techniques to Predict in Healthcare Case Study: Chronic Kidney Failure Disease", IJDMS, Vol.8, No.3, 2016, pp. 1-9.

[11] A. Salek and J. Stanko "Detection of Chronic Kidney Disease and Selecting Important Predictive Attributes", ICHI, Vol.8, 2016, pp. 1-9.

[12] RUEY .C, R. Y. CHEN and S. JEN "INTELLIGENT SYSTEMS ON THE CLOUD FOR THE EARLY DETECTION OF CHRONIC KIDNEY DISEASE", International Conference on Machine Learning and Cybernetics, Vol.8, 2012, pp. 1737-1742.

[13] C. Wu, R. Chiu and S. Wang "A cloud-based fuzzy expert system for the risk assessment of chronic kidney diseases", Int. J. Business and Systems Research, Vol. 9, No. 4, 2015, pp. 315-333.

[14] S. Nathaniel, A. Motwani and A. Saxena "Cloud based Predictive Model for Detection of 'Chronic Kidney Disease' Risk", International Journal of Computer Sciences and Engineering, Vol. 6, No. 4, 2018, pp. 185-188.

[15] A. Bat and V. Sin "A Review to Predictive Methodology to Diagnosis Chronic Kidney Disease", International Conference on Computing for Sustainable Global Development, IEEE, Vol.4, 2016, pp. 2760-2763. 